\title{
Modelling the spread and growth of Caulerpa taxifolia in closed waterways in southern Australia using cellular automata
}

\author{
L. McArthur, ${ }^{\text {a } J . ~ D u n n ~ a n d ~ S . ~ S c h r e i d e r ~}$ \\ a School of Mathematical and Geospatial Sciences, RMIT University, GPO BOX 2476, Melbourne, 3000 \\ Email: lynne.mcarthur@rmit.edu.au
}

\begin{abstract}
This study presents the mathematical development of a cellular automata model for the species Caulerpa taxifolia for closed or intermittently closed waterways along the Australian coast. The model is used to assess the spatial coverage of $C$. taxifolia by describing changes in growth, spread and total biomass for the species. Building upon a foundation model developed by the authors, this study was designed to enhance the predictive capabilities of a model based upon a discrete version of Laplace's equation. The improvements relate to several components integrated into the Laplacian coefficients; a periodic function which represents the seasonal variations in growth, the incorporation of the prevailing wind to represent the most likely direction of spread, and growth restrictions based on lake depth. The additional complexity improved the predictive capability of the model.
\end{abstract}

Cellular Automata (CA) have been used to model changing plant distributions over the last 20 years, providing efficient models for complex environmental systems, particularly of exotic species. In this project the discrete CA algorithm is designed to determine the state or biomass $B=\{-1,0,1,2\}$ of the current cell using the primary rule of the discrete Laplacian system. Biomass of -1 refers to land, and the other values represent the relative quantity of the weed in the cell; none, sparse or dense respectively. Cell interactions are governed by the coefficients of the Laplacian system which is discussed.

The foundation model incorporated simple rules, not unlike those of John Conway's Game of Life. The biomass of the surrounding cells at time $t$ determines the state of the central cell at time $t+1$. The boundary conditions were catered for by allocating a biomass of negative one to the land cells adjacent to the water.

The results indicate that the model is able to predict the total surface coverage and total biomass at levels of accuracy commensurate with the input data, which is important for control measures. Also, high accuracy in the predicted locational data at Lake Conjola indicates that the model is able to identify appropriate growing conditions to aid in the eradication efforts. At successive time steps, the model produces accurate patch location data with a slight overestimation on patch size due to slight error prediction of the decay in the initial winter season.

The principle objective of this new study is to improve the predictive capabilities of the model developed by the authors, by taking into account the biological and environmental factors of growth and spread and in doing so, more accurately predict the spatial coverage and colonization locations of $C$. taxifolia growth and spread in a closed or intermittently closed estuary. This model is designed to inform resource managers and government bodies of the most effective methods of eradication.

Keywords: Caulerpa taxifolia, cellular automata, biomass dynamics, Laplacian system 
McArthur et al., Modelling the spread and growth of Caulerpa taxifolia ...

\section{INTRODUCTION}

Non-indigenous, invasive, aquatic weeds significantly alter the ecosystems they colonize. This has been observed where the marine, green algae Caulerpa taxifolia, ((Vahl) C. Agardh) has been identified in many areas of the world including Europe, America and Australia (Meinesz, 1996; Withgott, 2002; Glasby et al., 2005). Fourteen waterways throughout New South Wales have infestations of $C$. taxifolia, typically located in the coastal estuaries (Creese et al., 2004). In New South Wales, C. taxifolia is classified as a Class 1 noxious species under the Fisheries Management Act, 1994 and as a marine "pest" in southern Australian waterways.

Two models of $C$. taxifolia have previously been developed: The first by Hill et al. (1998) who successfully modelled dispersal for the Mediterranean Sea invasion site using a coupled geographical information system - stochastic discrete event simulation model for large, open geographical areas with strong water currents. Secondly, the density-dependant rate of increase of $C$. taxifolia has also been modelled where a specific focus was placed on total biomass, in order to evaluate the success of various control management programs (Ruesink and Collado-Vides, 2006). In contrast, the current study focuses on small-scaled, closed or intermittently closed estuaries in shallow waterways and centers around predicting the likely spatial coverage and colonization locations of $C$. taxifolia.

Previous work by the authors produced a two-dimensional cellular automata model, based on the discrete Laplace equation, for Lake Conjola in NSW, Australia. This model was not designed to accurately predict $C$. taxifolia growth and spread; more to identify the most important drivers of the spread and growth patterns. The following factors were identified as the key indicators that were required for model improvement:

- Seasonal biomass fluctuations, in particular the depletion of biomass during winter, (Komatsu and Meinesz, 1997), and reduced probability of substrate attachment (Ruesink and Collado-Vides, 2006);

- Fragment recruitment from neighbouring cells due to currents (wind) (Wright and Davis, 2006);

- The incorporation of seagrass data to indicate improved likelihood of growth due to high nutrient levels and improved substrate structure for new colonisation (Wright and Davis, 2006);

- Depth limitations on growth: No growth has been observed at depths exceeding 10 metres at Lake Conjola;

- Anthropogenic influences on distribution: i.e. transference by boat or anchor (West et al., 2007).

This paper describes the more recent model which was developed to incorporate some of these factors. In particular, items 1 - 4 listed above. Item 5, the anthropogenic interference, has not been included due to lack of reliable data.

\subsection{Description of C. taxifolia}

Caulerpa taxifolia is a marine green seaweed (alga) that is endemic to tropical and sub-tropical regions around the world. In Australia, native populations of C. taxifolia are found in the Northern Territory, Queensland, Western Australia and on Lord Howe Island (Phillips and Price, 2002).

Outbreaks of an invasive strain of $C$. taxifolia were discovered in the cool waters around Monaco in 1984 and since that time the seaweed has spread rapidly throughout the Mediterranean. By the end of 2000, C. taxifolia was estimated to cover approximately $131 \mathrm{~km}^{2}$ of seafloor in the Mediterranean (Jaubert et al., 2003). The invasive strain in the Mediterranean was dubbed the 'aquarium strain' because it was derived from a strain cultivated for decoration in aquaria.

It is believed that $C$. taxifolia does not reproduce sexually, but rather spreads by growth and fragmentation the species is capable of regenerating from a small fragment of any part of the plant (Wright, 2005). However, this is true only for the invasive strain - sexual reproduction has been observed in some native populations.

Its biomass increases both by natural growth and by the spread of fragments that break off and colonize new locations. The transport of fragments is facilitated by currents, (in this study represented by wind), interaction with fishers and boaters and through other marine species physically interacting with the alga.

\subsection{Study Region}

The study uses data from Lake Conjola, Figure 1a. The data was collected by marine botanists, using GPS systems and personal observation. $C$. taxifolia in this waterway has been allowed to grow and spread without the imposition of eradication procedures, and is thus an ideal source of data to develop the model. A second estuary, the Narrawallee Inlet, is used to test the model's adaptability to other waterways. Lake Conjola and the 
a)

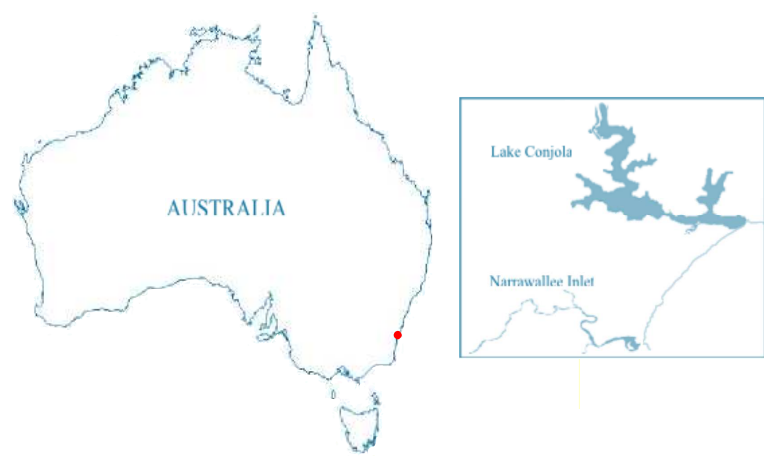

b)

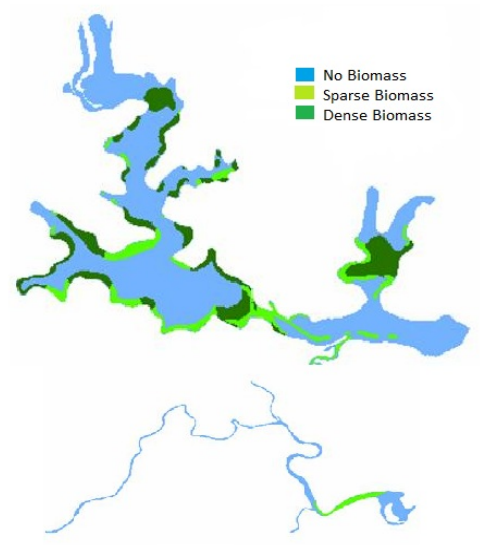

Figure 1. a) Map of study sites: Lake Conjola and the Narrawallee Inlet. b) Initial conditions in Lake Conjola (top) and the Narrawallee Inlet, March 2003.

Narrawallee Inlet are wave-dominated estuaries. The narrow entrances to the Pacific ocean of Lake Conjola and the Narrawallee Inlet mean that the volume of water exchanged during each tide is small and in dry conditions the entrances are often closed with no water exchanged, and the primary circulation is governed by wind driven currents. The study uses the prevailing wind direction as a proxy for current, and thus controls the direction of spread of the fragments. Figure $1 \mathrm{~b}$ indicates the extent of $C$. taxifolia invasion in both Lake Conjola and Narrawallee Inlet for March 2003.

\section{METHODS}

\subsection{Description of the Model}

The model uses a square gridcell representation of the region where the biomass or 'state' of each cell is contained in an $m \times n$ array. The biomass data are stored in a matrix of cells $\mathbf{B}$ where each cell $B_{x, y}, x=$ $1,2,3, \ldots, m, y=1,2,3, \ldots, n$, contains the recorded biomass at time $t, B_{x, y}(t)$. Here the indices $x$ and $y$ correspond to the longitude and latitude coordinate pair (the grid is scaled so that $\delta(x, y)=1$ ). Each cell in matrix $B$ contains biomass $B_{x, y}(t)$ at time $t$.

The discrete form, given by the finite difference scheme for the diffusion equation, can be formulated as:

$$
\begin{aligned}
\frac{\left[B_{x, y}(t+1)-B_{x, y}(t)\right]}{\delta t}= & \frac{a(t)\left[B_{x+\delta x, y}(t)-2 B_{x, y}(t)+B_{x-\delta x, y}(t)\right]}{\delta x^{2}} \\
& +\frac{a(t)\left[B_{x, y+\delta y}(t)-2 B_{x, y}(t)+B_{x, y-\delta y}(t)\right]}{\delta y^{2}},
\end{aligned}
$$

where $\delta x$ and $\delta y$ are spatial increments and $\delta t$ is the time step, in this study equivalent to 1 month (McArthur et al., 2006; Dunn et al., 2011).

The growth and spread was simulated using MATLAB, with the biomass in each cell being updated at each time step according to a set of rules. Equation (1) indicates that the biomass, $B$, at location $(x, y)$ at time $(t+1)$ is a linear combination of the biomass in that cell and the neighbouring cells at time $t$. Equation (1) can be rearranged to formulate the equation describing the change in biomass at each time step:

$$
\begin{aligned}
B_{x, y}(t+1)= & \alpha(t) B_{x, y}(t)+\beta_{x, y}\left[B_{x+\delta x, y}(t)+B_{x-\delta x, y}(t)\right. \\
& \left.+B_{x, y+\delta y}(t)+B_{x, y-\delta y}(t)\right]
\end{aligned}
$$

where $\alpha(t)$ is the growth function, which changes over time, and $\beta_{x, y}$ is the spread coefficient which changes over space; both of which can be expressed in terms of $a(t)$, (Dunn et al., 2011).

The model described here also includes contribution from diagonal cells, which simply implies that the rule for the Laplace operator works in linearly transformed coordinates based on the distance equivalence $\frac{\delta t}{\sqrt{\delta x^{2}+\delta y^{2}}}$ 
with $\delta(x, y, t)=1$ :

$$
\begin{aligned}
B_{x, y}(t+1)= & \alpha(t) B_{x, y}(t)+\beta_{x, y}\left[B_{x+\delta x, y}(t)+B_{x-\delta x, y}(t)\right. \\
& +B_{x, y+\delta y}(t)+B_{x, y-\delta y}(t)+\frac{1}{\sqrt{2}} B_{x-\delta x, y-\delta y}(t) \\
& \left.+\frac{1}{\sqrt{2}} B_{x-\delta x, y-\delta y}(t)+\frac{1}{\sqrt{2}} B_{x+\delta x, y+\delta y}(t)+\frac{1}{\sqrt{2}} B_{x+\delta x, y-\delta y}(t)\right] .
\end{aligned}
$$

The boundary condition is of Neumann type with no biomass spread from water to land and no biomass lost to outside the estuary. This seems feasible given that growth and fragmentation occurs solely during times of minimal to zero flow and when entrances are primarily closed. The growth is associated with diffusion, being the natural increase in biomass due to growth (and decrease due to decay) and spread is primarily related to new colonizations due to fragments of $C$. taxifolia breaking off and being transported either by human intervention or currents driven by wind. These two processes are catered for differently in the algorithm and for this reason, $\alpha(t)$ is expressed as time dependent, and $\beta_{x, y}$ spatially dependent.

\subsection{Algorithm}

Let the sum of the biomass in the neighbouring cells be given by:

$$
\begin{aligned}
S_{x, y}= & B_{x+\delta x, y}(t)+B_{x-\delta x, y}(t) \\
& +B_{x, y+\delta y}(t)+B_{x, y-\delta y}(t)+\frac{1}{\sqrt{2}} B_{x-\delta x, y-\delta y}(t) \\
& +\frac{1}{\sqrt{2}} B_{x-\delta x, y-\delta y}(t)+\frac{1}{\sqrt{2}} B_{x+\delta x, y+\delta y}(t)+\frac{1}{\sqrt{2}} B_{x+\delta x, y-\delta y}(t),
\end{aligned}
$$

then the growth and spread equation can be formulated as

$$
B_{x, y}(t+1)=\alpha(t) B_{x, y}(t)+\beta_{x, y} S_{x, y}(t),
$$

which indicates that the biomass in cell $B_{x, y}$ at time $t+1$ is governed by natural growth or decay at the rate given by $\alpha(t)$ and the spread from surrounding cells given the correct conditions described below, at the rate of $\beta_{x, y}$.

According to a set of rules the biomass in each cell fluctuates between 0 and $K=2$, the cell carrying capacity. The land cells, where $B=-1$ do not change, and are used in the algorithm to indicate the boundary. Fluctuations correspond to the growth rate, $\alpha(t)$, and the spread coefficient $\beta_{x, y}$. The biomass in a given cell in the next time-step is a function of the biomass currently in that cell, the biomass in the adjacent cells, the growth rate which is influenced by the presence or absence of seagrass, and the spread from adjacent cells, which is driven by the wind.

Throughout simulation, the biomass data is converted from categorical data, $B_{x, y} \in\{-1,0,1,2\}$, to continuous numerical data through application of the algorithm, and back to categorical data for output.

\subsection{Growth}

Two parameters, $p$ and $d$ are threshold values for equation (5) related to the sum of the biomass in the surrounding cells, $S$. Adjacency is defined by the degree of contact of the surrounding cells and includes all eight cells surrounding cell $B_{x, y}$. The time step, $t$, is month and model is initialized with $t_{0}$ representing March, 2003.

Growth occurs subject to $S_{x, y}>p$ and decay occurs subject to $S_{x, y} \leq d$. Given that plant growth is seasonally dependant we can describe $\alpha(t)$ by a cyclic function, given by equation (6) where $g$ is the amplitude of growth and $f(t)$ a time dependant sine function adjusted to coincide with $t_{0} \Rightarrow$ March 2003.

\subsection{Seagrass}

There is evidence to suggest that $C$. taxifolia frequently colonizes in seagrass habitats (Bell and Pollard, 1989), and in fact prefers those areas. For additional biomass in a given cell due to the presence of seagrass, the seagrass data for each waterway is stored in an $n \times m$ matrix of cells $O_{x, y}$, where each cell takes on a state 
according to $O_{x, y}=0,1$. The values 0 and 1 represent no seagrass and seagrass presence respectively. If cell $x, y$ contains seagrass, then the 'favourable growth condition' is applied. Combining these factors, the growth function can be defined as

$$
\alpha(t)=g * z(t)+\sigma O_{x, y}, \quad \text { where } \quad z(t)=\sin \left(\frac{(t+5) \pi}{6}\right)+0.4
$$

and $\sigma$ is the weighting factor for the favourable growth condition, and $g$ is the intrinsic growth rate.

\subsection{Spread}

Spread occurs when growth is positive, given that the surrounding cells have sufficient biomass, specified by the threshold $p$. Spread of $C$. taxifolia is due to fragmentation colonization, and in this model is driven by the frequency of the prevailing wind, since the dry conditions of the two estuaries during growth periods, $\alpha(t)>0$, suggest that the internal water circulation is driven primarily by wind (Wortmann, 1998). In the winter months, the biomass of $C$. taxifolia decreases, so there is no contribution from fragmentation nor growth.

Plant spread within estuaries is not uniform, so $\beta_{x, y}$ is defined as an array of coefficients governing the nonuniform dispersal of the plant to surrounding cells, based upon the relative frequency of wind direction. Wright and Davis (2006) observed that fragments of $C$. taxifolia do not disperse far from the originating cell due to negative buoyancy, so only fragmentation to immediate neighbours is included in the model. Thus, the final model, for $k, l \in\{-1,0,1\}$ is given by

$$
\begin{aligned}
B_{x, y}(t+1)= & \alpha(t) B_{x, y}(t)+\beta_{x+1, y} B_{x+1, y}(t)+\beta_{x-1, y} B_{x-1, y}(t) \\
& +\beta_{x, y+1} B_{x, y+1}(t)+\beta_{x, y-1} B_{x, y-1}(t) \\
& +\frac{1}{\sqrt{2}} \beta_{x-1, y+1} B_{x-1, y+1}(t)+\frac{1}{\sqrt{2}} \beta_{x-1, y-1} B_{x-1, y-1}(t) \\
& +\frac{1}{\sqrt{2}} \beta_{x+1, y+1} B_{x+1, y+1}(t)+\frac{1}{\sqrt{2}} \beta_{x+1, y-1} B_{x+1, y-1}(t), \\
\text { where } \beta= & \beta \text { if } \alpha(t)>0 \\
= & 0 \text { if } \alpha(t)<0 .
\end{aligned}
$$

\section{RESULTS}

The observed data for Lake Conjola and the Narrawallee Inlet confirm that there was a significant decay in the total surface coverage of $C$. taxifolia over the June to September period in both 2003 and 2004. Over the March 2003 to August 2003 period at Lake Conjola the decrease in total surface coverage was 7.374 hectares. At the smaller Narrawallee Inlet, the decay in total surface coverage was from $5970 \mathrm{~m}^{2}$ in March 2003 to $190 \mathrm{~m}^{2}$ in August 2003. Decay in total biomass in both estuaries was also recorded over the same period. Observed data indicated that there was an $11 \%$ decline in total biomass at Lake Conjola and at Narrawallee Inlet this decline was $97 \%$.

Close correspondence between the simulated and observed data for Lake Conjola, especially the total number of cells containing biomass, Figure $2 \mathrm{a}$ and the total biomass, Figure $2 \mathrm{c}$ is evident. There is a discrepancy between the observed and simulated data at the August 2003 time step for the total biomass due to an underestimation in the decay of the total biomass for the time when $\alpha(t)<0$. A similar trend is observed at the Narrawallee Inlet. The lower decay rate in the simulation led to higher rates in the total number of invaded cells, Figure $2 \mathrm{~b}$ and the total biomass, Figure $2 \mathrm{~d}$, at successive timesteps, even with a reasonably accurate growth rate for $\alpha(t)>0$.

The graphs for the validation site, Narrawallee Inlet, indicate diversion of the simulated results for both the number of cells occupied and the total amount of biomass from the observed data. This would suggest that there are factors at this site that have not been taken into account. Perhaps the Inlet is influenced more by exogenous factors such as rainfall and temperature, or by the shape and depth of the waterway. These are topics for further study.

Seasonal trends in the model and the observed series are quite similar, only with less decay during $\alpha(t)<0$ in the simulated data. Noticeably, the Narrawallee Inlet results show that the decay in biomass in each successive winter is less extreme than the previous winter. This trend is consistent with that of the observed data, where 

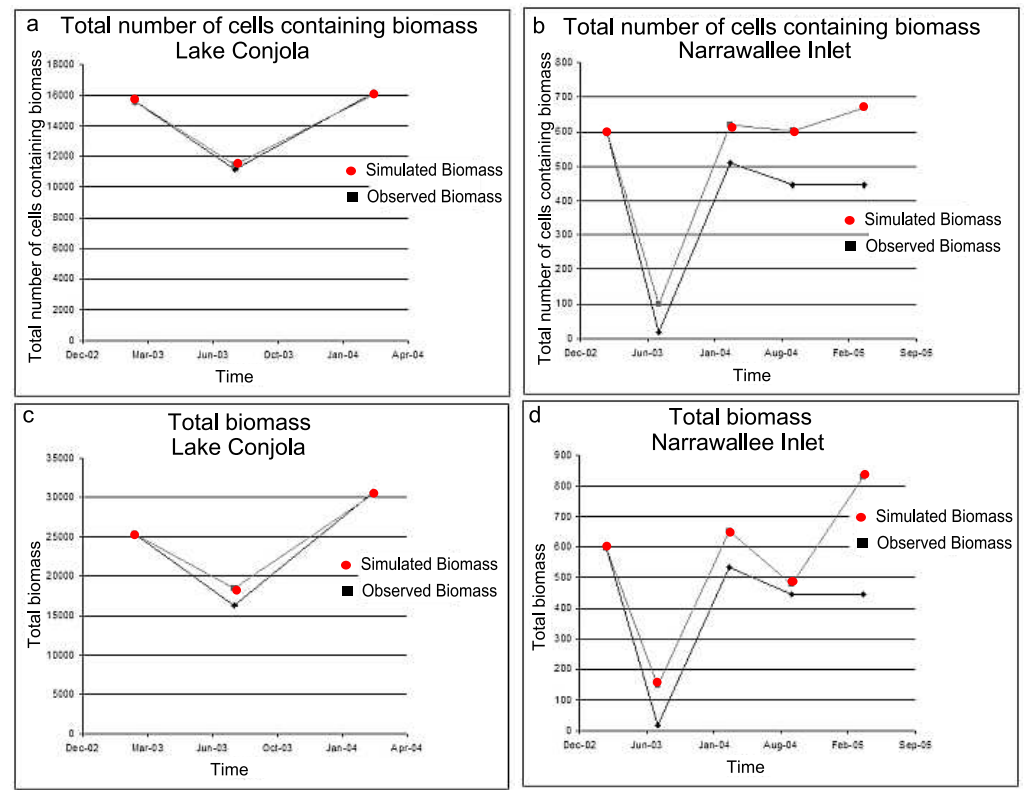

Figure 2. a. Comparison of the total number of cells containing biomass at Lake Conjola over a 12 month period from March 2003 to March 2004. b. Comparison of the total number of cells containing biomass at the Narrawallee Inlet over a 25 month period from March 2003 to April 2005. c. Comparison of the total biomass at Lake Conjola over a 12 month period from March 2003 to March 2004. d. Comparison of the total biomass at the Narrawallee Inlet over a 25 month period from March 2003 to April 2005.

the decrease in decay rates can be attributed to the increase in the density of biomass in each cell and sparse areas of growth (the areas that completely decay over the winter period) are rare. This again indicates that the modelled behaviour of $C$. taxifolia compares well with the observed behaviour.

The comparison of the location of $C$. taxifolia for March 2004 at Lake Conjola yields close association between the biomass matrices. There is a slight overestimation of biomass in patches near the edge of the waterway and significant variation in the north-east of the waterway. This variation can be attributed to the assigned growth limitation in the model. Limitations in this case lead to loss of detail in the shallow areas of the waterway particularly evident in the north-east corner. The other area of noticeable deviation is the central region in the North-East when a depletion of growth has formed in the observed data set. Speculation regarding this phenomenon includes deepening of the area from flooding or formation of islands due to drought, with the second more likely.

\section{CONCLUSION}

The increased complexity of the model by the introduction of the biological and geographical properties has resulted in the model taking on a sense of realism. The results indicate that the model describes the growth and spread of $C$. taxifolia in Lake Conjola very well according to the simple metrics developed. Simulation of C. taxifolia in the Narrawallee Inlet gives a good indication of spread compared with observed data indicating the models adaptability to different waterways. The application of the model to the significantly smaller Narrawallee Inlet indicates that even though there are variations in later time steps the model clearly identifies suitable growth locations.

Discrepancy between actual and predicted biomass for this waterway is suspected to be attributed to the fluctuating flow rates in this particularly shallow waterway which results in exposure of the estuary bed on frequent occasions. Further improvements in the model would include depth data, which would provide addition of growth in each cell as a function of the depth and the water current rather than limiting spread to occur within a distance of the edge of the waterway which is used as a proxy for depth. Additionally, nutrient properties need to be explored for their effect on the growth and decay of C. taxifolia.

There are potential problems with accuracy due to the optimisation and interpretation of parameters. In particular, the increase in growth due to seagrass presence represented by $\alpha$, and the limitation on the carrying 
McArthur et al., Modelling the spread and growth of Caulerpa taxifolia ...

capacity $K$. The foundation model provided a means of identifying the most significant biological and environmental factors that were necessary for improving the model's predictability, while ensuring that the model remained parsimonious and not overly complex. The seasonal fluctuations and spread by fragmentation, produced significant improvement in modelling results and hence in the predictive capabilities of the model.

\section{REFERENCES}

Bell, J. D. and D. A. Pollard (1989). Ecology of fish assemblages and fisheries associated with seagrasses. In A. Larkum, A. McComb, and S. Shepherd (Eds.), Biology of seagrasses: A treatise on the biology of seagrasses with special reference to the Australian region, pp. 565-609. Amsterdam: Elsevier.

Creese, R., A. Davis, and T.M.Glasby (2004, June). Eradicating and preventing the spread of the invasive alga Caulerpa taxifolia in NSW. NSW Fisheries Final Report Series 64, NSW Fisheries.

Dunn, J., L. McArthur, and S. Schreider (2011). Population and nutrient dynamics in aquatic ecosystems: modelling blooming phenomenon. In F. Chan, D. Marinova, and R. Anderssen (Eds.), MODSIM2011, 19th International Congress on Modelling and Simulation, pp. 3698-3704.

Glasby, T., P. Gibson, and S. Kay (2005). Tolerance of the invasive marine alga Caulerpa taxifolia to burial by sediment. Aquatic Botany 82, 71-81.

Hill, D., P. Coquillard, J. de Vaugelas, and A. Meinesz (1998). An algorithmic model for invasive species: Application to Caulerpa taxifolia (Vahl) C. Agardh development in the North-West Mediterranean Sea. Ecological Modelling 109, 251-265.

Jaubert, J., J. Chisholm, A. Minghelli-Roman, M. Marchioretti, J. Morrow, and H. Ripley (2003). Reevaluation of the extent of Caulerpa taxifolia development in the northern Mediterranean using airborne spectrographic sensing. Marine Ecology Progress Series 263, 75-82.

Komatsu, T. and D. Meinesz, A.and Buckles (1997). Temperature and light responses of the alga Caulerpa taxifolia introduced into the Mediterranean Sea. Marine Ecology Press Series 146, 145-153.

McArthur, L., S. Schreider, T. Glasby, and A. Alizadeh Shabani (2006). Modelling the spread of Caulerpa taxifolia in Lake Conjola, Southern NSW. In M. Hamza (Ed.), Proceedings of the 15th IASTED International Conference on Applied Simulation and Modelling, pp. 1-3.

Meinesz, A. (1996). Killer Algae. Chicago, USA: University of Chicago Press.

Phillips, J. and I. Price (2002). How different is Mediterranean Caulerpa taxifolia (Caulerpales: Chlorophyta) to other populations of the species? Marine Ecology Progress Series 238, 61-71.

Ruesink, J. and L. Collado-Vides (2006). Modelling the increase and control of Caulerpa taxifolia - an invasive marine microalga. Biological Invasions 8, 309-325.

West, E., P. Barnes, J. Wright, and A. Davis (2007). Anchors aweigh: Fragment generation of invasive Caulerpa taxifolia by boat anchors and its resistance to desiccation. Aquatic Botany 87(3), 196-202.

Withgott, J. (2002). California tries to rub out the monster of the lagoon. Science 295, 2201-2202.

Wortmann, J. (1998). Evalutation the effects of freshwater inflow on the distribution of estuarine macrophytes. Ecological Modelling 106, 213-232.

Wright, J. (2005). Differences between native and invasive Caulerpa taxifolia: a link between asexual fragmentation and abundances in invasive populations. Marine Biology 147(2), 559-569.

Wright, J. and A. Davis (2006). Demographic feedback between clonal growth and fragmentation in an invasive seaweed. Ecology 87(7), 1744-1754. 